\title{
Intrabiliary Acetylcysteine Infusion for Biliary Obstruction in a Case of Chronic Pancreatitis with Pancreatic Head IPMN
}

\author{
Paul K Vincent ${ }^{1}$ Hasim Ahamed ${ }^{1}$ Rajesh Gopalakrishna, ${ }^{1, \odot}$ Ramachandran Menon ${ }^{1}$ Rama P. Venu ${ }^{1}$ \\ ${ }^{1}$ Department of Gastroenterology, Amrita Institute of Medical \\ Address for correspondence Dr. Rajesh Gopalakrishna, MD, DNB \\ Sciences, Ponekkara, Cochin, Kerala, India \\ (Gastro), MNAMS, FICP, Department of Gastroenterology, Amrita Institute \\ of Medical Sciences, Amrita Viswa Vidyapeetham, Ponekkara, Kochi, \\ Kerala 682 041, India (e-mail: grajesh22@gmail.com).
}

\begin{abstract}
Keywords

- acetylcysteine

- cholangitis

- IPMN

- chronic pancreatitis

Successful biliary drainage in case of intraductal papillary mucinous neoplasm (IPMN) with thick mucus by ERCP and internal drainage alone is usually inadequate. The mucolytic properties of acetylcysteine can offer a therapeutic benefit in this setting. We report a 50-year-old man with chronic pancreatitis and IPMN who had recurrent episodes of biliary obstruction due to mucous occlusion, despite multiple stent exchanges. A nasobiliary catheter was placed and infusion of acetylcysteine was performed which resulted in significant improvement of jaundice. He later underwent surgery after nutritional rehabilitation. This case report highlights the role of intrabiliary acetylcysteine infusions for mucous occlusions in IPMN.
\end{abstract}

\section{Introduction}

Intraductal papillary mucinous neoplasms (IPMNs) are papillary neoplasms within the main pancreatic duct showing mucin hypersecretion, leading to dilatation of the pancreatic duct. They are uncommon pancreatic neoplasms with malignant potential. We report a case of chronic pancreatitis in a patient in whom main duct IPMN was detected on pancreatoscopy and he was successfully managed by incorporating preoperative intrabiliary acetylcysteine infusion.

\section{Case Report}

A 50-year-old male, who had been diagnosed with chronic pancreatitis since 5 years and was on pancreatic enzymes replacement therapy, presented to us with abdominal pain, decreased appetite, and jaundice. Laboratory investigations showed a neutrophilic predominant leukocytosis and cholestatic jaundice (total bilirubin of $23.5 \mathrm{mg} / \mathrm{dL}$ ) with a direct fraction of $16 \mathrm{mg} / \mathrm{dL}$, AST (aspartate aminotransferase) of $351 \mathrm{IU} / \mathrm{mL}$, ALT (alanine aminotransferase) of $276.2 \mathrm{IU} / \mathrm{mL}$, and ALP (alkaline phosphatase) of $340 \mathrm{IU} / \mathrm{mL}$. MDCT (multi detector computed tomography) abdomen showed a multicystic mass involving the head and uncinate process and communicating with pancreatic duct and measuring $4.6 \mathrm{~cm} \times 5.3 \mathrm{~cm} \times 6 \mathrm{~cm}$. It showed $180^{\circ}$ encasement of portal vein. Atrophy of pancreatic body and tail with dilated pancreatic duct and multiple pancreatic ductal calculi in the distal body and tail region were noted. Marked biliary dilatation was also noted. Duodenoscopy ( - Fig. 1) showed a patulous ampulla with mucus plug and mucous extruding from orifice ("fish mouth" appearance). Pancreatogram showed a dilated pancreatic duct and pancreatoscopy ( - Fig. 2) performed using Spy glass system (Boston Scientific, Spencer, IN, USA), showed a dilated duct with calculi and large quantities of mucinous material and papillary projections, suggestive of IPMN ("fish egg" appearance). As his cholangiogram showed a markedly dilated CBD (common bile duct), and possibility of stent migration was high, we placed a $7 \mathrm{Fr} 7 \mathrm{~cm}$ double pigtail plastic stent (Cook) in the CBD. As he continued to have worsening jaundice and fever spikes, a second ERCP (endoscopic retrograde cholangiopancreatography) was done after 2 days. Blocked stent in situ was noted which was removed using snare. Balloon sweeps after a 5 to $7 \mathrm{~mm}$ sphincterotomy revealed copious amounts of mucinous material. A $10 \mathrm{Fr} 7 \mathrm{~cm}$ double pigtail stent (COOK) was deployed into the CBD in the hope that this would ensure adequate biliary drainage. As he continued to have 


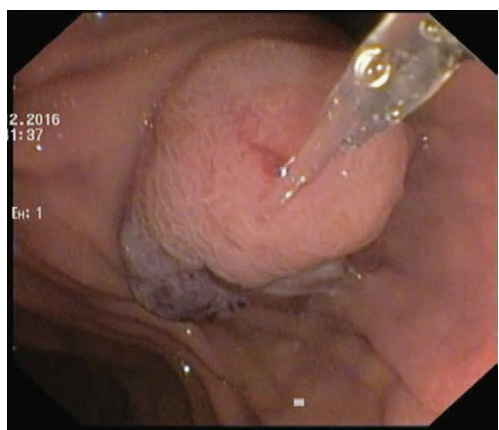

Fig. 1 "Fish mouth" appearance of ampulla on duodenoscopy.

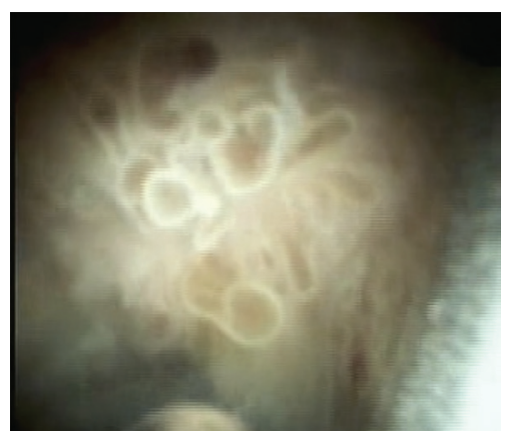

Fig. 2 “Fish egg" appearance on pancreatoscopy using Spyglass system.

cholangitis, a third ERCP was done after 5 days from the second ERCP. Mucus plug was noted at ampulla and previous stent appeared to be plugged with mucus. A $10 \mathrm{Fr}$ nasobiliary drain (COOK) placed in the $\mathrm{CBD}$ and $\mathrm{N}$-acetylcysteine (NAC) was instilled (4,000 mg NAC in $500 \mathrm{~mL} \mathrm{NS}) ; 100 \mathrm{~mL}$ of NAC was flushed every 3 hours. He was also continued on broad-spectrum IV (intra venous) antibiotics. With these measures we noticed a steady decline in the hyperbilirubinemia and our patient reported clinical improvement of symptoms. However, his symptoms were not completely resolved. He underwent open cholecystectomy with placement of a T-tube through which infusion of acetylcysteine was continued while he was in the hospital for an earlier resolution before definitive surgery. Subsequently, he was discharged and continued on nutritional rehabilitation. On follow-up after 1 week, his liver function tests had normalized. He was subsequently taken up for Whipple's resection. The resected specimen showed intraductal papillary mucinous neoplasm of pancreas (intestinal type) with low- and high-grade dysplasia and microinvasion, against a background of severe chronic pancreatitis. He was asymptomatic at follow-up of over 1 year.

\section{Discussion}

World Health Organization (WHO) defines IPMNs as intraductal mucin-producing neoplasms with tall, columnar, mucin-containing epithelium with or without papillary projections. What differentiates IPMNs from other mucinous cystic neoplasms is the lack of the ovarian stroma. Based on histology, IPMNs are classified into benign (adenoma), borderline, or malignant (carcinoma in situ or invasive).
Clinically, patients may present with history of abdominal pain, nausea, steatorrhea, and weight loss, and may mimic chronic pancreatitis. Rarely may they coexist together in same patient as in our case. Surgical resection is the preferred treatment in IPMN with high-grade dysplasia or invasive malignancy. Prognosis after resection is good with 5-year disease-specific survival of at least $75 \%$. A consensus guideline for the management of IPMN was established in $2006 .{ }^{1}$

Radiological cross-sectional imaging (magnetic 3 imaging [MRI] with magnetic resonance cholangiopancreatography [MRCP] or computed tomography [CT]) are useful for diagnosis and preoperative staging. Additional evaluation with endoscopic ultrasound (EUS) with fine-needle aspiration (FNA) may be needed to confirm a diagnosis or to assess for malignant features. Pancreatoscopy and direct visualization of the pancreatic duct is very sensitive for making a diagnosis, especially in the main duct type. The patulous pancreatic orifice can be cannulated easily with a pancreatoscope during ERCP, permitting direct visualization of the pancreatic duct. Pancreatoscopy can help determine the extent of IPMN, especially if skip lesions are present, which may aid preoperative staging. Features suggesting malignancy include spotty or linear red markings, "fish egg"-like lesions, villous proliferations, and vegetative-type lesions.

The uses of acetylcysteine (NAC) in health care are manifold. Its mucolytic action is utilized in the management of chronic obstructive pulmonary disease and idiopathic pulmonary fibrosis. ${ }^{2,3}$ It also has antioxidant and anti-inflammatory properties and is a very safe agent. Intrabiliary use of acetylcysteine for advanced biliary IPMN has been reported in recent case reports. ${ }^{4}$ Acetylcysteine can be used in palliative setting as well as preoperatively (as in our case) to decrease the tenacious mucinous secretion in IPMN where biliary drainage alone by stenting alone proves inadequate.

Our case shows the rare occurrence of the radiological features of chronic pancreatitis and endoscopic evidence of intraductal papillary mucinous neoplasm. Our case also demonstrates successful use of a novel approach to reduce cholestatic jaundice in IPMN patients by the intrabiliary instillation of NAC prior to definitive surgical resection.

\section{Conflict of Interest}

None declared.

\section{References}

1 Tanaka M, Fernández-del Castillo C, Adsay V, et al; International Association of Pancreatology. International consensus guidelines 2012 for the management of IPMN and MCN of the pancreas. Pancreatology 2012;12(3):183-19710.1016/j.pan.2012.04.004

2 Demedts M, Behr J, Buhl R, et al. High-dose acetylcysteine in idiopathic pul- monarybrosis. $N$ Engl J Med 2005;353:2229-224210.1056/NEJMoa042976

3 Sadowska AM. N-acetylcysteine mucolysis in the management of chronic obstructive pulmonary disease. Ther Adv Respir Dis 2012;6(3):127-135

$4 \mathrm{Hu} \mathrm{LH}$, Liu MH, Liao Z, et al; Continuous infusion of $\mathrm{N}$-acetylcysteine by nasobiliary for advanced intraductal papillary mucinous neoplasm of bile ducts (with video). Am J Gastroenterol 2012;107(12):1929-1930 